\title{
Influence of Childhood Scleroderma on Physical Function and Quality of Life
}

\author{
EILEEN M. BAILDAM, HOLLY ENNIS, HELEN E. FOSTER, LINDSAY SHAW, ALICE S.E. CHIENG, JANE KELLY, \\ ARIANE L. HERRICK, and HELEN L. RICHARDS
}

\begin{abstract}
Objective. There have been few studies of quality of life in childhood scleroderma and these focused predominantly on self-perception and the influence of skin lesions. Our cross-sectional study aimed to describe the influence of childhood scleroderma on physical function and quality of life in relation to clinical and demographic measures.

Methods. Children with either localized scleroderma or systemic sclerosis (SSc) attending pediatric rheumatology clinics, together with their parents or guardians, were asked to complete a set of 4 validated measures. Clinical and demographic data were provided by consultant pediatric rheumatologists. Results. In total, 28 children and their parents/guardians participated in the study (68\% female, median age 13 yrs; $86 \%$ localized scleroderma, $14 \%$ SSc). The median Child Health Assessment Questionnaire (CHAQ) score was 0.1 (range 0-3, 0 indicating no impairment), the median Child Dermatology Life Quality Index (CDLQI) score was 5 (range 0-30, 0 indicating no impairment), and the median Child Quality of Life Questionnaire (CQOL) function score was 26 (range 0-105, 0 indicating no impairment). Family activity, measured by the Child Health Questionnaire (CHQ-PF50), was also moderately impaired by scleroderma, with a median score of $83(0-100,100$ indicating no impairment).

Conclusion. Scleroderma had only a moderate effect on quality of life and physical function as measured by the 4 validated instruments. Although a small number of children reported greater impairment, this is an encouraging finding, given its potential disfiguring and debilitating effects. (First Release Nov 1 2010; J Rheumatol 2011;38:167-73; doi:10.3899/jrheum.100447)
\end{abstract}

Key Indexing Terms:

QUALITY OF LIFE

PHYSICAL FUNCTION

CHILDHOOD-ONSET SCLERODERMA

Childhood scleroderma represents a rare and poorly understood spectrum of conditions. Localized scleroderma, including "morphea," predominantly affects the skin and

From the Alder Hey Children's NHS Foundation Trust, Liverpool, Arthritis Research UK Epidemiology Unit, University of Manchester, Manchester Academic Health Science Centre, Manchester;

Musculoskeletal Research Group, Newcastle University, and Newcastle Hospitals NHS Trust, Newcastle-upon-Tyne; Bristol Dermatology Centre, Bristol Royal Infirmary, Bristol; Royal Manchester Children's Hospital, Manchester, UK; and Department of Clinical Health Psychology, Mercy University Hospital, Cork, Republic of Ireland.

Supported by the Raynaud's and Scleroderma Association.

E.M. Baildam, FRCP, Consultant Paediatric Rheumatologist, Alder Hey Children's NHS Foundation Trust; H. Ennis, PhD, Research Associate, Arthritis Research UK Epidemiology Unit, University of Manchester. Manchester Academic Health Science Centre; H.E. Foster, FRCP, Professor in Paediatric Rheumatology, Musculoskeletal Research Group, Newcastle University, and Newcastle Hospitals NHS Trust; L. Shaw, MRCP, Consultant Dermatologist, Bristol Dermatology Centre, Bristol Royal Infirmary; A.S.E. Chieng, MRCP, Consultant Paediatric Rheumatologist, Royal Manchester Children's Hospital; J. Kelly, BSc, Specialist Paediatric Rheumatology Nurse, Alder Hey Children's NHS Foundation Trust; A.L. Herrick, FRCP, Reader in Rheumatology, Arthritis Research UK Epidemiology Unit, University of Manchester, Manchester Academic Health Science Centre; H.L. Richards, PhD, Lead Clinical Psychologist,

Department of Clinical Health Psychology, Mercy University Hospital.

Address correspondence to Dr. E.M. Baildam, Alder Hey

Children's NHS Foundation Trust, Liverpool, UK.

E-mail:Eileen.Baildam@alderhey.nhs.uk

Accepted for publication August 6, 2010. subcutaneous tissue and accounts for the majority of childhood scleroderma ${ }^{1,2,3,4}$. We recently reported its incidence in childhood in the UK and Ireland to be 3.4 cases per million per year ${ }^{5}$. Scleroderma occurring as part of the multisystem connective tissue disease systemic sclerosis (SSc) is even rarer $^{6,7}$, with an estimated 0.27 per million children per year affected in the $\mathrm{UK}^{5}$. Childhood scleroderma is a heterogeneous disease with considerable variation in terms of disease severity, the location of lesions, and the extent of disability and disfigurement. Although rare, it can be associated with significant morbidity in those affected.

Health-related quality of life is now a recognized measure of disease effect and there is a rapidly expanding literature devoted to quality of life in childhood-onset disease. Although there are several studies of quality of life in adult populations with scleroderma ${ }^{8,9,10,11,12}$, childhood and adolescent-onset scleroderma have received less attention. The focus of studies to date has been on the influence of skin lesions on quality of life and self-perception ${ }^{13,14}$, and there has been relatively little discussion of the role of physical function, pain, family relations, or social limitations. Tools are nevertheless required to measure outcome in trials of treatment, and quality of life issues are likely to be part of a composite assessment of cases to measure disease activity and to estimate disease effects. 
We report a cross-sectional study that describes quality of life and physical function in a cohort of children with scleroderma and their association with clinical and demographic characteristics, using a range of published and validated instruments. We hypothesized that children's quality of life and physical function would be impaired by scleroderma, with additional specific objectives as follows: (1) To assess whether impairment varies according to disease subtype, the location of skin lesions, and musculoskeletal involvement in localized scleroderma. (2) To detect if there are any differences between child and parent/guardian assessments of impairment. (3) To estimate the impact of childhood scleroderma on families.

\section{MATERIALS AND METHODS}

Participants. Between May 2007 and July 2008, children aged between 4 and 16 years with a diagnosis of scleroderma confirmed by a consultant rheumatologist, and attending rheumatology clinics in tertiary referral centers at Alder Hey Children's Hospital (Liverpool), Royal Manchester Children's Hospital (Manchester), Royal Victoria Infirmary (Newcastle), and the Royal National Hospital for Rheumatic Diseases (Bath) were invited to participate, together with their parents.

Procedure. After giving informed, written consent, participants (children and parents) were asked to complete the questionnaires detailed below. Demographic and clinical information was provided for all children by consultant pediatric rheumatologists or dermatologists. The study was approved by the North West Research Ethics Committee.

Questionnaires. Four self-report questionnaires, validated for childhood physical and psychiatric disease, were used to assess physical and social function and quality of life, and were completed by children or parents as appropriate. Respondents were asked to reflect specifically on the influence of their scleroderma. Assessments were also made of illness perceptions of scleroderma, to be reported at a later date. Opportunity was also given for participants to provide additional free-text comments and these are reported below.

Physical function and pain. The Childhood Health Assessment Questionnaire (CHAQ) ${ }^{15}$ : The CHAQ is a pediatric modification of the Stanford Health Assessment Questionnaire $(\mathrm{HAQ})^{16}$ shown to be a valid and sensitive tool in the evaluation of physical function in children with chronic arthritis ${ }^{15}$. The scoring and range of possible scores (0-3) are the same as the HAQ, with higher scores indicating greater impairment. It also includes a visual analog scale (VAS) for pain with scores 0-100. It was completed by children aged 11 years or more and by parents of children aged 10 years and younger.

Quality of life based on effects of skin lesions. Children's Dermatology Life Quality Index (CDLQI) ${ }^{17}$ : The CDLQI was developed as a specific tool to assess quality of life in children with skin conditions and was designed for children aged 4 to 16 years. An illustrated version of the CDLQI was shown to be equivalent to the written version, but easier and faster for children to $u^{u} \mathrm{e}^{18}$. The CDLQI consists of 10 questions and generates a score of 0-30, higher scores indicating greater impairment, and contains 6 subdomains: (1) Symptoms and feelings, (2) Leisure, (3) School and holidays, (4) Personal relationships, (5) Sleep, and (6) Treatment.

Quality of life based on function and emotional response. Child Health-related Quality of Life (CQOL) ${ }^{19}$ : The CQOL is a generic measure designed to assess quality of life in children with chronic physical disorders, psychiatric disorders, and learning difficulties. Both parents and children were asked to rate function over the past month on a 7-point Likert scale across 15 different domains. "Upset" and "satisfaction" levels for each domain are gauged with identical scales. Combined scores are computed by adding the rating of the 15 domains, higher scores indicating greater impairment, higher levels of upset, and the least satisfaction.
Parent assessment and social function. Child Health Questionnaire (CHQ-PF50) ${ }^{20}$ : The CHQ-PF50 is a generic measure that assesses a child's physical, emotional, and social well-being from the perspective of parents, and is scored 0-100. Lower scores indicate greater impairment and higher levels of emotional distress. Physical domains include physical function, social limitations caused by physical health, bodily pain/discomfort, and general health. Psychosocial domains include social limitations caused by emotional well-being, behavior, mental health, and self-esteem. Four items documenting the effects of a child's illness on parents and family life include emotional effect on parents, influence on parent's time, limitations on family activities, and effects on family cohesion.

Clinical assessments. A modified Paediatric Rheumatology European Society (PRES) form was used to collect demographic and clinical data ${ }^{4,7}$. For each child, consultants were asked to document age, sex, ethnicity, date of disease onset, and extent and location of skin lesions using this form and the treatments currently received, including methotrexate, steroids (parenteral or oral), physiotherapy, and occupational therapy. Cases were classified according to subtype as localized scleroderma or SSc. Data were also collected on location of skin lesions in localized scleroderma.

Analysis. The principal aim of the study was hypothesis-generating. Data were analyzed using Stata software (Version 9.2). The quality of life questionnaires were scored as published in accompanying manuals and literature. The distribution of data suggested the use of nonparametric tests of significance and Wilcoxon Mann-Whitney U tests and Spearman's correlation coefficients were calculated as appropriate. Differences between parent and child CQOL subscales were calculated as discrete variables where both copies were available. For the CHAQ, questionnaires completed by children and parents were to be treated similarly if there were no significant differences in scores. Clinical and demographic variables assessed were gender, age at assessment, disease duration since onset, disease subtype, and location of skin lesions in localized scleroderma.

\section{RESULTS}

Demographic information. Thirty children and their parents agreed to participate in the study (97\% of those approached) and completed the questionnaires. Two cases fell outside the study age range (4-16 yrs) and were excluded. In 2 cases, participants only marginally exceeded the upper age limit of 16 years (by 2 weeks and by 2 months) and were included within the study. Characteristics of the 28 participants are shown in Table 1 . The majority of the sample was female $(n=19)$. Disease type, age at onset, and age at diagnosis were similar in males and females.

The number of children and parents completing each questionnaire is shown in Table 2.

Clinical characteristics. The majority of children (24/28) had localized scleroderma. Of these 24, $15(63 \%)$ had linear scleroderma (including 3 with en coup de sabre), 5 (21\%) had plaque morphea, and 4 (16\%) had both linear scleroderma and plaque morphea. Joint contractures were present in 4 children (3 linear scleroderma, 1 morphea) and 3 children had arthritis (1 linear scleroderma and 2 morphea). The locations of lesions are reported in Table 3 . The remainder $(4 / 28)$ had limited cutaneous $\mathrm{SSc}^{21}$ with skin thickening distal to the elbows, knees, and neck. All 4 had Raynaud's phenomenon, 3 had sclerodactyly, skin induration, and musculoskeletal involvement, and 2 had gastrointestinal involvement. The majority of children $(>96 \%)$ received some form of treatment at time of assessment, principally methotrexate 
Table 1. Demographic and clinical features of the 28 patients with scleroderma, including by subgroup.

\begin{tabular}{lccc}
\hline Characteristic & $\begin{array}{c}\text { Total Sample, } \\
\mathrm{n}=28\end{array}$ & $\begin{array}{c}\text { Localized, } \\
\mathrm{n}=24\end{array}$ & $\begin{array}{c}\text { SSc, } \\
\mathrm{n}=4\end{array}$ \\
\hline Female, $\mathrm{n}(\%)$ & $19(68)$ & $15(63)$ & $4(100)$ \\
Caucasian, n (\%) & $24(86)$ & $20(87)$ & $4(100)$ \\
Age at assessment, median (range), yrs & $13(5-17)$ & $13(5-17)$ & $11.7(7-14)$ \\
Disease duration since diagnosis, median (range), mo & $30(2-135)$ & $22(2-135)$ & $68(15-83)$ \\
Methotrexate, $\mathrm{n}(\%)$ & $22(79)$ & $21(88)$ & $1(25)$ \\
Parenteral steroids, n (\%) & $9(32)$ & $9(38)$ & 0 \\
Physiotherapy, $\mathrm{n}(\%)$ & $10(36)$ & $7(29)$ & $3(75)$ \\
Occupational therapy, n (\%) & $4(14)$ & $2(8)$ & $2(50)$ \\
CHAQ physical function score, median (range), 0-3 & $0.1(0-1.6)$ & $0(0-1.6)$ & $0.6(0.1-1.2)$ \\
CHAQ VAS pain score, median (range), 0-100 & $5(0-85)$ & $0(0-85)$ & $12.5(10-55)$ \\
CDLQI total score, median (range), 0-30* & $5(0-10)$ & $5(0-10)$ & $3(0-6)$ \\
CQOL function score, children, median (range), 0-105** & $26(10-53)$ & $26(10-53)$ & $40(36-44)$ \\
CQOL function score, parents, median (range), 0-105*** & $28(13-64)$ & $26(13-64)$ & $40(27-54)$ \\
\hline
\end{tabular}

* 23 cases (localized scleroderma $\mathrm{n}=19, \mathrm{SSc} \mathrm{n}=4$ ). ** 21 cases (localized scleroderma $\mathrm{n}=19, \mathrm{SSc} \mathrm{n}=2$ ). $* * * 25$ cases (localized scleroderma $\mathrm{n}=21, \mathrm{SSc} \mathrm{n}=4$ ). CHAQ: Childhood Health Assessment Questionnaire; CDLQI: Child Dermatology Life Quality Index; CQOL: Child Quality of Life Questionnaire.

Table 2. Results for questionnaire completion.

\begin{tabular}{lccc}
\hline Questionnaire & Completed by Children, $\mathrm{n}$ & $\begin{array}{c}\text { Completed by Parents on } \\
\text { Behalf of Children }\end{array}$ & Completed by Both \\
\hline CHAQ & 18 & 10 & - \\
CDLQI & $27^{*}$ & - & - \\
CQOL & 21 & 25 & - \\
CHQ-PF50 & 27 & - & 19 \\
\hline
\end{tabular}

* The CDLQI was completed by 27 children. An overall CDLQI was available for 23 cases who completed all questions. CHAQ: Childhood Health Assessment Questionnaire; CDLQI: Child Dermatology Life Quality Index; CQOL: Child Quality of Life Questionnaire. CHQ-PF50: Child Health Questionnaire.

Table 3. Questionnaire scores according to lesion location in localized scleroderma.

\begin{tabular}{|c|c|c|c|c|}
\hline Measure & $\begin{array}{c}\text { Total Sample, } \\
\mathrm{n}=24\end{array}$ & $\begin{array}{c}\text { Trunk/Limb Lesions, } \\
\mathrm{n}=14\end{array}$ & $\begin{array}{c}\text { Head/Face Lesions, } \\
n=9\end{array}$ & $\begin{array}{l}\text { Trunk/Limb and Face } \\
\text { Lesions, } \mathrm{n}=1\end{array}$ \\
\hline CHAQ physical function score, median (range), $0-3$ & $0.1(0-1.6)$ & $0.1(0-1.6)$ & $0(0-1)$ & 0 \\
\hline CDLQI total score, median (range), $0-30^{*}$ & $5(0-10)$ & $5(0-7)$ & $1(0-9)$ & 10 \\
\hline CQOL function score, children, median (range), $0-105$ & $26(10-53)$ & $30(15-50)$ & $20(13-64)$ & 42 \\
\hline CQOL function score, parents, median (range), $0-105$ & $28(13-64)$ & $26(17-44)$ & $19(10-53)$ & 40 \\
\hline
\end{tabular}

* 19 cases. CHAQ: Childhood Health Assessment Questionnaire; CDLQI: Child Dermatology Life Quality Index; CQOL: Child Quality of Life Questionnaire. VAS: visual analog sale.

(22/28), parenteral steroids $(9 / 28)$, and physiotherapy (10/28). Of the 4 children with SSc, one received methotrexate and prednisolone and the remainder received one or more of calcium channel blockers, angiotensin-converting enzyme inhibitors, and vitamin D.

Physical function and pain: CHAQ. No significant difference was detected between parent and child scores and data were treated in the same way. Median CHAQ and VAS pain assessments for the overall sample and by disease subtype are shown in Table 1 and by skin lesion location in Table 3 . A statistically significant difference was detected between the underlying distributions of CHAQ scores by subtype $(\mathrm{z}=-1.97, \mathrm{p}=0.04)$, with the SSc group reporting higher scores indicating greater physical impairment. In children with localized scleroderma, median scores were higher for those with trunk and/or limb lesions than those with facial lesions ( 0.1 vs 0 , respectively), but this difference was not statistically significant. Median VAS pain scores were high-

Personal non-commercial use only. The Journal of Rheumatology Copyright (c) 2011. All rights reserved. 
er in children with SSc than in those with localized scleroderma (12.5 vs 0 ), and among those with localized disease, were higher in those with trunk and/or limb lesions than in those with facial lesions (5 vs 0 ), but these differences were not statistically significant. There was a positive correlation between CHAQ scores and VAS pain scores for the overall sample $(\mathrm{p}=0.6, \mathrm{p}<0.001)$, with the greatest physical impairment more likely to be reported by those with the highest pain ratings (Figure 1). No further relationship was detected with other clinical and demographic variables.

Quality of life based on the effect of skin lesions: CDLQI. Median scores (on a scale of 0-30) for the overall sample and by disease subtype are shown in Table 1 . The localized disease group had a higher median score than the SSc group (5 vs 3 , respectively) and children with localized trunk and/or limb lesions had a higher median score than those with facial lesions (5 vs 1 ). The highest score of 10 was for a case involving lesions to the trunk, limbs, and face. These differences were not statistically significant and no association was detected between either the total score or individual subscales and clinical and demographic variables.

Quality of life based on function and emotional response: CQOL. No significant difference was detected between overall parent and child scores. Cumulative scores for child and parent ratings of physical function (from a maximum of 105) are shown in Table 1. Median differences between child and parent scores were calculated in 19 cases where both copies were available. This produced a median difference of 8 (range -33 to 90 ) in favor of parents for the combined score and by subscale: 3 (range -17 to 67 ) for satisfaction, 3 (range -36 to 25 ) for function, and 1 ( -16 to 24 ) for upset, with parents reporting higher scores in each. There was a positive correlation between differences in global scores and age at assessment $(\mathrm{p}=0.4, \mathrm{p}=0.03)$. In other words, the difference between parent and child scores increased significantly with children's age at assessment. No association was found between differences in subscale scores and the clinical and demographic variables.

Parent assessment and social function: CHQ-PF50. Median scores by disease subtype are shown in Figure 2, with high scores indicating minimal impairment. Median scores for physical domains in the SSc group were $\leq 83$, with the lowest scores for items "bodily pain" (50) and "general health" (54). Median scores for the localized group were 100 for physical domains, with the exception of "general health" (64). Within psychosocial domains, scores in the localized group indicated greater impairment than in the SSc group, with the exception of "behavior" (68 in the SSc group vs 73 in the localized group). Scores for "impact on parent's time" indicated only minimal disturbance (100 for the localized group and 94 for the SSc group). Greater disturbance was indicated by the scores for "emotional impact on parents" (67 for the localized group, 75 for the SSc group), "family activity" (85 for the localized group, 56 for the SSc group), and "family cohesion" ( 85 for the localized group, 73 for the SSc group). No association was detected between CHQ scores and the clinical and demographic variables.

Free-text comments. Sixteen parents provided additional comments about their children on the CQOL questionnaire (57\% of the sample). Six comments were positive (example, "my child is doing well and I hope it continues" and my child has "continued with daily activities"). Ten parents commented upon negative aspects and these can be divided into 4 themes: medication or hospital appointments causing difficulties (mentioned by 3 ), child self-conscious of physical problems caused by scleroderma (mentioned by 4 ), child

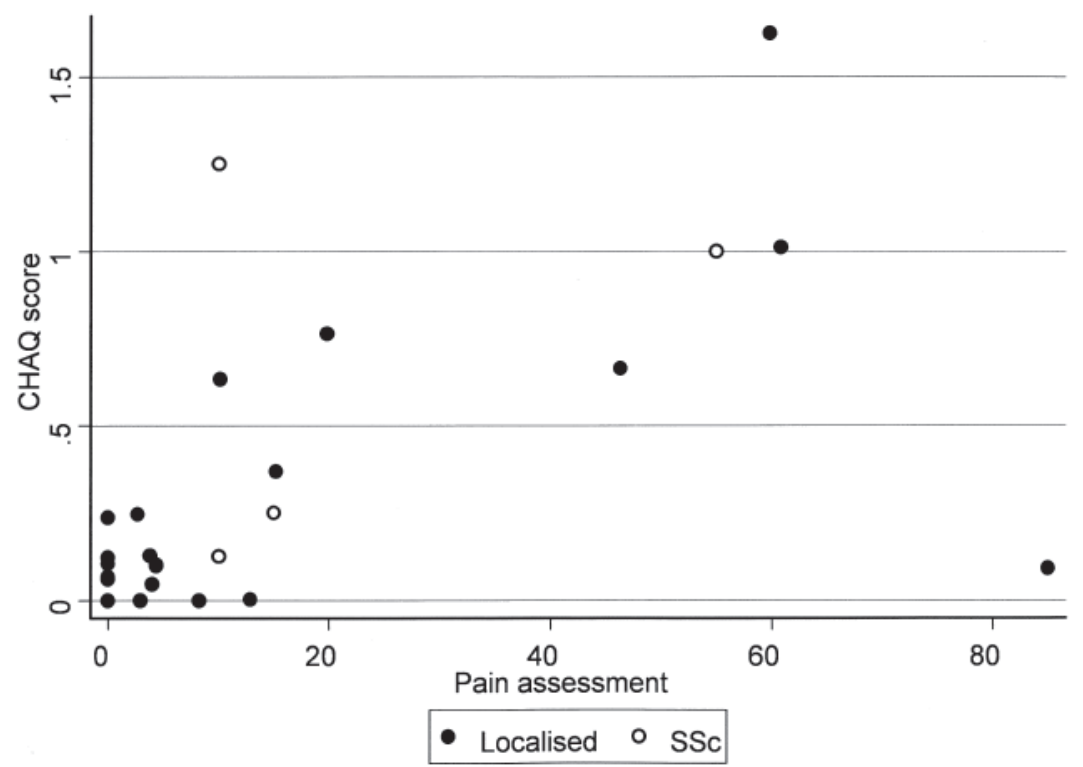

Figure 1. VAS pain scores (0-100) and CHAQ physical function scores in children with localized scleroderma and SSc. 


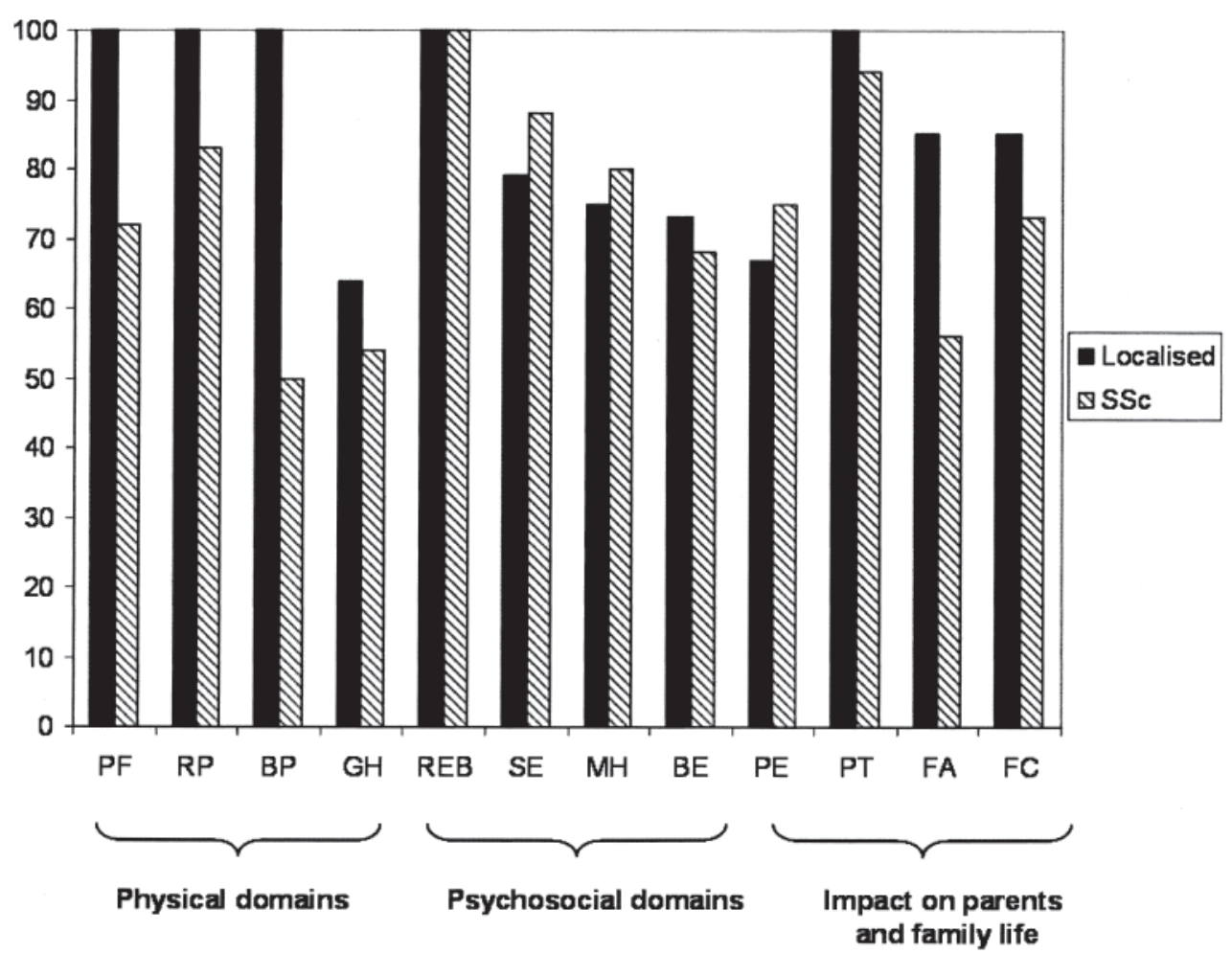

Figure 2. Median CHQ-PF50 item and domain scores comparing localized and SSc groups. PF: physical function; RP: social limitations caused by physical health; BP: bodily pain/discomfort; GH: general health; REB: social limitations caused by emotional well-being; SE: self-esteem; MH: mental health; BE: behavior; PE: emotional effects on parents; PT: effects on parents' time; FA: family activities; FC: family cohesion.

having difficulty relating to peers or siblings (mentioned by 2), and child having difficulty doing physical exercise and/or pain following physical exercise (mentioned by 3 ). Children with trunk or limb lesions accounted for $7 / 10$ of the negative comments and only $1 / 6$ of the positive comments.

\section{DISCUSSION}

The study set out to determine if children with scleroderma had impaired quality of life or physical function, and whether this impairment varied according to clinical and demographic variables including sex, age, disease subtype, and location of skin lesions. The results have shown that only a moderate impairment to quality of life and physical function was seen in the majority of children within our sample, although a small number reported much higher levels of impairment.

We used 4 validated questionnaires to assess quality of life and physical function. The CDLQI, a skin disease-specific measure, was used by Orzechowski, et al ${ }^{14}$ in a cohort of children with localized scleroderma: these investigators found only moderate impairment with a median score of $2^{14}$. The children in our cohort had a median score of 5 (which remained 5 when the SSc cases were excluded), indicating moderate impairment comparable to the reference ranges for children with relatively minor skin conditions such as acne
$(0.7-10)$ or localized eczema $(0-10)^{22}$. The highest score of 10 was for a child with localized scleroderma of the trunk, limbs, and face, yet, in keeping with a previous study examining self-perception in a cohort of children with morphea, children with localized facial lesions did not have significantly higher levels of impairment and there was no association between impairment and the age of the child ${ }^{13}$.

The 3 additional questionnaires focused specifically on physical function (CHAQ), the influence of chronic physical disease on everyday life (CQOL), and family life from the perspective of parents or caregivers (CHQ-PF50) to determine if the physical and emotional burden of scleroderma adversely affected quality of life. The majority of the sample $(17 / 28)$ had a CHAQ score $<0.1$, indicating little impairment, 8 children had CHAQ scores between 0.2 and 0.3 , comparable with reference ranges for children with juvenile pauciarticular arthritis $(0.22-0.34)^{15}$, and 3 had scores of between 1 and 1.6, comparable to the range for children with systemic juvenile arthritis $(1.2-1.7)^{15}$. Children with SSc had significantly higher scores than those with localized scleroderma, but differences according to localized skin lesions were not statistically significant. Pain scores were higher in those with greater physical function impairment, although the highest score of 85 was in a case with trunk lesions and a relatively low CHAQ score. With regard to the CQOL, physical function was rated by parents Personal non-commercial use only. The Journal of Rheumatology Copyright (c) 2011. All rights reserved. 
$(n=25)$ as within the reference range for children with chronic physical disease (15.3-35.7) ${ }^{19}$ in 14 cases, as better than this in 1 case, and as worse than this in 9 cases (range 39-64). Parents reported higher CQOL scores than children (indicating greater impairment), and these differences increased significantly for older age groups but were not associated with any demographic or clinical data collected in the study. It is possible that, as children grow older, parents become more aware of quality of life impairment in light of school performance, career prospects, or life chances and this may influence responses.

In terms of the influence of scleroderma on parents and family life, the greatest impairment in CHQ-PF50 subscale scores appeared to be in physical domains, particularly bodily pain and general health, for children with SSc, and in psychosocial domains for children with localized scleroderma, particularly self-esteem, mental health, and the effects on parents' emotions. Scleroderma, and most notably SSc, was perceived to have a detrimental influence on family activity, with scores lower than the reference range for a normal US child population $(71-100)^{23}$ in $11 / 28$ cases. This does suggest that childhood scleroderma may have a significant effect on parents and families and that the emotional consequences of living with scleroderma are important. This aspect was highlighted by the free-text comments provided by some parents that indicated several areas of concern, particularly in relation to children with localized lesions affecting the trunk or limbs.

Our study has several limitations. The rarity of childhood scleroderma and the convenience sampling employed in the data collection imposed some limits on interpretation of the data. All patients were recruited from tertiary referral centers and, therefore, represent pediatric specialist practice rather than general pediatric experience. Moreover, the requirement to recruit from 4 collaborating centers meant that it was not possible for the same researcher to meet all the families. Therefore standardized questionnaires were utilized to collect data in contrast to a semistructured interview approach. As a single cross-sectional measurement, it is also possible that there would be change across the disease course and over time, and a followup assessment may have detected these changes. Another potential limitation of the study was the lack of a control group to compare with the scleroderma sample. However, we have endeavored to compare our results with published reference ranges wherever possible.

A composite measure assessing the extent and activity of localized lesions, such as the recently developed "localized scleroderma severity scale" 24,25 , have proved useful but no such measure was available when this study began. Clinical and demographic data were instead collected using the modified PRES form, a questionnaire previously used within epidemiological studies to provide detailed information about subtype, clinical features, and the location of skin involvement. The PRES form also allowed us to verify cases from different centers, an important point given the lack of consensus over the classification of childhood scleroderma.

Despite these limitations, our study does suggest, in keeping with previous studies, that childhood scleroderma has a moderate influence on quality of life in terms of physical appearance and location of skin lesions and on physical function and family life. Childhood scleroderma does differ from adult-onset disease: mortality rates in childhood SSc are lower than in adult populations and the longterm outcomes in terms of mortality are more favorable than for adult-onset disease ${ }^{1,26}$. However, there is also an increased incidence of overlap syndromes in childhood-onset scleroderma, the potential for growth problems, and an increased use of immunosuppressive therapies in the management of childhood scleroderma ${ }^{1}$. This study does serve to emphasize the heterogeneity of childhood scleroderma and the range that exists in terms of how children are physically affected and the influence upon their quality of life. Explaining the variance that exists in terms of how children cope with the emotional consequences of their condition is more complex. It is possible that the therapy that children within this population received, particularly physiotherapy and occupational therapy, may have helped them to cope with the condition. The actions and explanations of clinical staff, family, and other individuals within children's lives are likely to have played a crucial role in helping children to manage scleroderma. This was outside the remit of our study; additional work was conducted to gauge illness perceptions and this will be reported elsewhere.

\section{REFERENCES}

1. Zulian F. Systemic sclerosis and localized scleroderma in childhood. Rheum Dis Clin North Am 2008;34:239-55.

2. Marzano A, Menni S, Parodi A, Borghi A, Fuligni A, Fabbri P, et al. Localized scleroderma in adults and children. Clinical and laboratory investigations of 239 cases. Eur J Dermatol 2003;13:171-6.

3. Christen-Zaech S, Hakim M, Sule Afsar F, Paller A. Pediatric morphoea (localized scleroderma): Review of 136 patients. J Am Acad Dermatol 2008;59:385-96.

4. Zulian F, Athreya BH, Laxer R, Nelson AM, Feitosa de Oliveira SK, Punaro MG, et al. Juvenile localised scleroderma: clinical and epidemiological features in 750 children. An international study. Rheumatology 2006;45:614-20.

5. Herrick A, Ennis H, Bhushan M, Silman A, Baildam E. Incidence of linear scleroderma and systemic sclerosis in the UK and Ireland. Arthritis Care Res 2010;62:213-8.

6. Foeldvari I. Scleroderma in children. Curr Opin Rheumatol 2002;14:699-703.

7. Martini G, Foeldvari I, Russo R, Cuttica R, Eberhard A, Ravelli A, et al. Systemic sclerosis in childhood. Clinical and immunological features of 153 patients in an international database. Arthritis Rheum 2006;54:3971-8.

8. Richards H, Herrick A, Griffin K, Gwilliam P, Fortune D. Psychological adjustment to systemic sclerosis - exploring the association of disease factors, functional ability, body-related attitudes and fear of negative evaluation. Psychol Health Med 
2004;9:29-39

9. Rannou F, Poiraudeau S, Berenze A, Baubet T, Le-Guern V, Cabane $\mathrm{J}$, et al. Assessing disability and quality of life in systemic sclerosis: construct validities of the Cochin Hand Function Scale, Health Assessment Questionnaire (HAQ), systemic sclerosis HAQ and Medical Outcomes Study 36-Item Short Form health survey. Arthritis Care Res 2007;57:94-102.

10. Del Rosso A, Boldrini M, D’Agostino D, Placidi GPA, Scarpato A, Pignone A, et al. Health-related quality of life in systemic sclerosis as measured by the Short Form 36: Relationship with clinical and biologic markers. Arthritis Care Res 2004;51:475-81.

11. Valentini G, Matucci-Cerinic M. Disease-specific quality indicators, guidelines and outcome measures in scleroderma. Clin Exp Rheumatol 2007;25:159-62.

12. Hudson M, Thombs B, Steele R, Panopalis P, Newton E, Baron M. Quality of life in patients with systemic sclerosis compared to the general population and patients with other chronic conditions. J Rheumatol 2009;36:768-72.

13. Uziel Y, Laxer R, Krafchik B, Rae S, Yeung M, Feldman B. Children with morphoea have normal self-perception. J Paediatr 2000; $137: 727-30$

14. Orzechowski N, Davis D, Mason T, Crowson C, Reed A. Health-related quality of life in children and adolescents with juvenile localized scleroderma. Rheumatology 2009;48:670-2.

15. Singh G, Athreya B, Fries J, Goldsmith D. Measurement of health status in children with juvenile rheumatoid arthritis. Arthritis Rheum 1994;37:1761-9.

16. Fries J, Spitz P, Kraines R, Holman H. Measurement of patient outcomes in arthritis. Arthritis Rheum 1980;23:137-45.
17. Lewis-Jones M, Finlay A. The Children's Dermatology Life Quality Index (CDLQI): Initial validation and practical use. Br J Dermatol 1995;132:942-9.

18. Holme S, Man I, Sharpe J, Dykes P, Lewis-Jones M, Finlay A. The Children's Dermatology Life Quality Index: validation of the cartoon version. Br J Dermatol 2003;148:285-90.

19. Graham P, Stevenson J, Flynn D. A new measure of health-related quality of life for children: preliminary findings. Psychol Health 1997; 12:655-65.

20. Landgraf J, Abetz L, Ware J. The CHQ user's manual. Boston: The Health Institute, New England Medical Centre; 1996.

21. LeRoy E, Medsger T. Criteria for the classification of early systemic sclerosis. J Rheumatol 2001;28:1573-6.

22. Beattie P, Lewis-Jones M. A comparative study of impairment of quality of life in children with skin disease and children with other childhood diseases. Br J Dermatol 2006;155:145-51.

23. Raat H, Bonsel G, Essink-Bot M, Landgraf J, Gemke R. Reliability and validity of comprehensive health status measures in children: the Child Health Questionnaire in relation to the Health Utilities Index. J Clin Epidemiol 2002;55:67-76.

24. Arkachaisri T, Pino S. Localized scleroderma severity index and global assessments: a pilot study of outcome instruments. J Rheumatol 2008;35:650-7.

25. Arkachaisri T, Fertig N, Pino S, Medsger T. Serum autoantibodies and their clinical associations in patients with childhood and adult onset linear scleroderma. A single center study. J Rheumatol 2009;35:2439-44.

26. Denton C, Derrett-Smith E. Juvenile onset systemic sclerosis: children are not small adults. Rheumatology 2009;48:96-7. 